\title{
RELATIVISTIC FIELD THEORY OF UNSTABLE PARTICLES
}

\author{
A. SALAM and P. T. MATTHEWS ${ }^{(*)}$
}

Imperial College, London

I feel quite ashamed not to be speaking about any indefinite metric. I am going to confine my remarks to the old familiar relativistic local field theory and discuss the problem of creating a framework for the description of unstable elementary particles. I need not apologize for this. Of the sixteen known elementary particles, only four - the proton, the electron, the neutrino and the photon are stable. The problem which exercised us was the problem of the construction of a state vector in a relativistic field theory corresponding to an unstable elementary particle.

Let me briefly recapitulate what one normally does in order to describe stable particles. Particles are characterized first of all by eigenstates of the energy momentum vector

$$
P_{\mu}\left|p_{\mu}, n\right\rangle=p_{\mu}\left|p_{\mu}, n\right\rangle .
$$

Here $p_{\mu}{ }^{2}$ is the mass of the particle and this is necessarily real. The further characterization of the state vectors is provided in general by specifying whether we are dealing with in-states or out-states. This needs an asymptotic description of these particles at plus or minus infinity of time in the conventional fashion. I shall denote these state vectors in this form :

$$
\left|k_{1} \ldots k_{N}\right\rangle_{\text {in }} \text { or }\left|k_{1} \ldots k_{N}\right\rangle_{\text {out }} .
$$

One then postulates that this set of states, both the instates as well as the out-states, satisfy the completeness relation which I shall write in the following form :

$$
\Sigma\left|k_{1} \ldots k_{N}\right\rangle \underset{\text { in }}{\text { out out }} \text { in }\left\langle k_{1} \ldots k_{N}\right|=1 .
$$

It is clear that for an unstable particle there is no asymptotic limit in any sense, and it is impossible to use this conventional procedure to construct or define any state whatever for this particle.

How does conventional theory cope with this? Consider a $\Sigma$-particle, which can decay into two stable $\chi$-particles $k_{1}$ and $k_{2}$ :

$$
k_{1}^{2}=k_{2}{ }^{2}=m^{2} \text {. }
$$

One assumes the existence of a $\Sigma$-particle state $\left|M^{0}\right\rangle$ and calculates the transition rate

$$
\lambda=\left|\left\langle M^{0} \mid k_{1} k_{2}\right\rangle\right|^{2}
$$

as if all particles were stable. Then the attenuation of a beam of such particles is given by

$$
d n / d \tau=-\lambda n(\tau)
$$

where $\tau$ is the proper time.

This may be interpreted as a wave function

$$
\langle\tau \mid \Phi\rangle=e^{i M^{0} \tau-\lambda|\tau| / 2} .
$$

The particle then appears with a complex mass : $M^{0}+i \lambda / 2$, which is certainly not consistent with (1), any more than the states $\left|M^{0}\right\rangle$ or $|\Phi\rangle$ belong in the completeness relation.

We wish to propose a restatement of this phenomenological description of long-lived elementary particles, which is consistent with the general scheme described in (1) and (2).

The Hilbert space of field theory is spanned completely by the stable particle states $\left|k_{1} k_{2} \ldots\right\rangle$. To describe unstable particles we define a density matrix

$$
\begin{gathered}
\varrho=\sum\left|k_{1} k_{2}\right\rangle \frac{\varrho\left(m^{2}\right)}{m^{2}}\left\langle k_{1} k_{2}\right|, \\
m^{2}=\left(k_{1}+k_{2}\right)^{2} .
\end{gathered}
$$

$\varrho\left(m^{2}\right)$ is essentially the probability of finding the $\Sigma$-particle with mass - or $Q$-value -- corresponding to $m$.

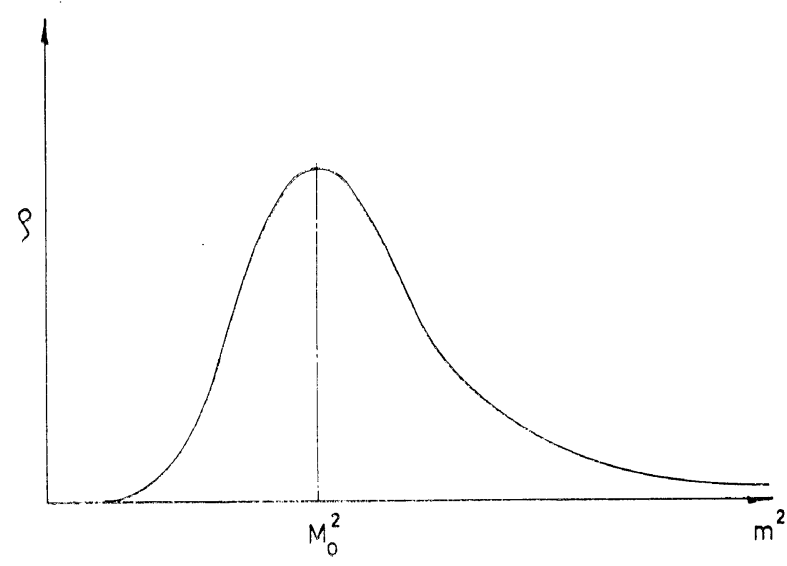

*) Reported by A. Salam 
The mean mass is then given by

$$
M^{0^{2}}=\frac{\operatorname{Tr} P_{\mu}^{2} \varrho}{\operatorname{Tr} \varrho}=\frac{\int m^{2} \varrho\left(m^{2}\right) d m^{2}}{\int \varrho\left(m^{2}\right) d m^{2}} .
$$

The decay constant is given by the spread in mass values, as determined by the second moment

$$
\left(M^{0} \lambda\right)^{2}=\frac{\operatorname{Tr}\left(\left(P_{\mu}^{2}\right)^{2}-\left(M^{0^{2}}\right)^{2}\right) \varrho}{\operatorname{Tr} \varrho} .
$$

In order to relate these definitions to field theory we assume the existence of a field $\Phi(x)$, satisfying the causality condition

$$
[\Phi(x), \Phi(y)]=0 \quad \text { for } \quad(x-y)^{2}<0 .
$$

We then assume

$$
\varrho\left(\left(k_{1}+k_{2}\right)^{2}\right)=\left|\left\langle 0|\Phi(0)| k_{1} k_{2}\right\rangle\right|^{2} .
$$

If the $\Phi$-field has to satisfy a field equation of the form

$$
(\square-\alpha) \Phi=F[\chi],
$$

then a general theorem due to Källén, Gell-Mann and Low and Lehman, states that

$$
\alpha=\frac{\int m^{2} \varrho\left(m^{2}\right) d m^{2}}{\int \varrho\left(m^{2}\right) d m^{2}} .
$$

Thus :

$$
\alpha=M^{02}
$$

as defined above.

For $F[\chi]$ we may assume :

$$
F[\chi]=g \chi^{2} .
$$

If $\varrho$ is computed by summing perturbation theory we obtain

$$
\varrho\left(m^{2}\right)=\frac{\lambda\left(m^{2}\right)}{\left(m^{2}-M^{02}\right)^{2}+\lambda^{2}\left(m^{2}\right)} .
$$

where $\lambda$ is the $\Sigma-2 \chi$ vertex part and $\lambda\left(M^{0}\right)$ gives the conventionally computed life-time to all orders. Clearly, the second moment (suitably defined) is $\lambda\left(\mathrm{M}^{0}\right)$, giving us in this approximation, the conventional result.

I must mention that Peierls has conjectured that the one particle propagator

$$
G\left(k^{2}\right)=\int \frac{\varrho\left(M^{2}\right)}{k^{2}-M^{2}} d M^{2}
$$

has a pole on the "unphysical sheet" which gives the lifetime and mean mass of an unstable particle.

\section{DISCUSSION}

Wataghin: I want to remark that, in a recent paper on non-local theories, I discussed the virtual states in a manner similar to that of Salam. It will be published in Nuovo Cimento ${ }^{1)}$. I introduced decaying virtual states of bare particles in a non-local field theory, where an explicit reference to the proper time and the c.m. system of interacting particles is made.

Low: It seems to me that in your approach the probability of decaying with some energy is, in fact, given by the density function. According to this, there should therefore be a finite probability, say, for a $A^{\circ}$ decaying into a proton and two pions.

Gell-Mann: Let us pursue this point a little further. You say, of course, that you want to consider only those states into which the particle can actually decay. Now let us consider, as Low did, the case of the $\Lambda^{\circ}$ decaying into a proton and a pion. Do we consider all energies of the final state? You did not put in any cut-off on the mass.

Salam: Let us consider this case (Fig. 1) where there are two humps. Now this is a standard case of two resonances in nuclear theory and one has the standard problem of resolving cleanly the two resonances. I have no solution to this question. There is the very simple case (Fig. 2) where you first have a sharp hump, then a very little tail and then a large second hump. But supposing one is dealing with cases where all the humps have the same sort of widths, then you may well ask to which particle does the region between the peaks belong. I do not know. I am pointing out a matter of principle, that from this point of view there is no distinction between the peaks, and they have all to be treated on the same footing.

Gell-Mann: Then I cannot agree that this is a very good definition. Let us consider the $\Lambda^{\circ}$ - particle once more. Let me call attention to the fact that if you turn off the

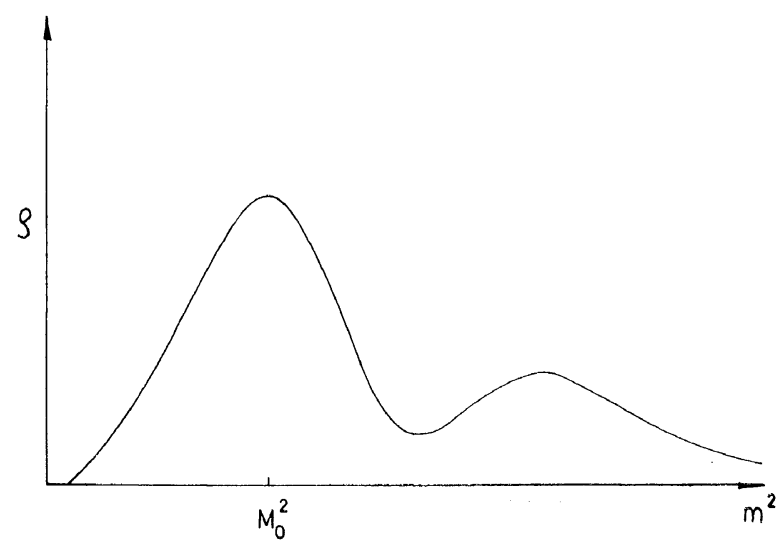

Fig. 1. 
The mean mass is then given by

$$
M^{0^{2}}=\frac{\operatorname{Tr} P_{\mu}^{2} \varrho}{\operatorname{Tr} \varrho}=\frac{\int m^{2} \varrho\left(m^{2}\right) d m^{2}}{\int \varrho\left(m^{2}\right) d m^{2}} .
$$

The decay constant is given by the spread in mass values, as determined by the second moment

$$
\left(M^{0} \lambda\right)^{2}=\frac{\operatorname{Tr}\left(\left(P_{\mu}^{2}\right)^{2}-\left(M^{0^{2}}\right)^{2}\right) \varrho}{\operatorname{Tr} \varrho} .
$$

In order to relate these definitions to field theory we assume the existence of a field $\Phi(x)$, satisfying the causality condition

$$
[\Phi(x), \Phi(y)]=0 \quad \text { for } \quad(x-y)^{2}<0 .
$$

We then assume

$$
\varrho\left(\left(k_{1}+k_{2}\right)^{2}\right)=\left|\left\langle 0|\Phi(0)| k_{1} k_{2}\right\rangle\right|^{2} .
$$

If the $\Phi$-field has to satisfy a field equation of the form

$$
(\square-\alpha) \Phi=F[\chi],
$$

then a general theorem due to Källén, Gell-Mann and Low and Lehman, states that

$$
\alpha=\frac{\int m^{2} \varrho\left(m^{2}\right) d m^{2}}{\int \varrho\left(m^{2}\right) d m^{2}} .
$$

Thus :

$$
\alpha=M^{02}
$$

as defined above.

For $F[\chi]$ we may assume :

$$
F[\chi]=g \chi^{2} .
$$

If $\varrho$ is computed by summing perturbation theory we obtain

$$
\varrho\left(m^{2}\right)=\frac{\lambda\left(m^{2}\right)}{\left(m^{2}-M^{02}\right)^{2}+\lambda^{2}\left(m^{2}\right)} .
$$

where $\lambda$ is the $\Sigma-2 \chi$ vertex part and $\lambda\left(M^{0}\right)$ gives the conventionally computed life-time to all orders. Clearly, the second moment (suitably defined) is $\lambda\left(\mathrm{M}^{0}\right)$, giving us in this approximation, the conventional result.

I must mention that Peierls has conjectured that the one particle propagator

$$
G\left(k^{2}\right)=\int \frac{\varrho\left(M^{2}\right)}{k^{2}-M^{2}} d M^{2}
$$

has a pole on the "unphysical sheet" which gives the lifetime and mean mass of an unstable particle.

\section{DISCUSSION}

Wataghin: I want to remark that, in a recent paper on non-local theories, I discussed the virtual states in a manner similar to that of Salam. It will be published in Nuovo Cimento ${ }^{1)}$. I introduced decaying virtual states of bare particles in a non-local field theory, where an explicit reference to the proper time and the c.m. system of interacting particles is made.

Low: It seems to me that in your approach the probability of decaying with some energy is, in fact, given by the density function. According to this, there should therefore be a finite probability, say, for a $A^{\circ}$ decaying into a proton and two pions.

Gell-Mann: Let us pursue this point a little further. You say, of course, that you want to consider only those states into which the particle can actually decay. Now let us consider, as Low did, the case of the $\Lambda^{\circ}$ decaying into a proton and a pion. Do we consider all energies of the final state? You did not put in any cut-off on the mass.

Salam: Let us consider this case (Fig. 1) where there are two humps. Now this is a standard case of two resonances in nuclear theory and one has the standard problem of resolving cleanly the two resonances. I have no solution to this question. There is the very simple case (Fig. 2) where you first have a sharp hump, then a very little tail and then a large second hump. But supposing one is dealing with cases where all the humps have the same sort of widths, then you may well ask to which particle does the region between the peaks belong. I do not know. I am pointing out a matter of principle, that from this point of view there is no distinction between the peaks, and they have all to be treated on the same footing.

Gell-Mann: Then I cannot agree that this is a very good definition. Let us consider the $\Lambda^{\circ}$ - particle once more. Let me call attention to the fact that if you turn off the

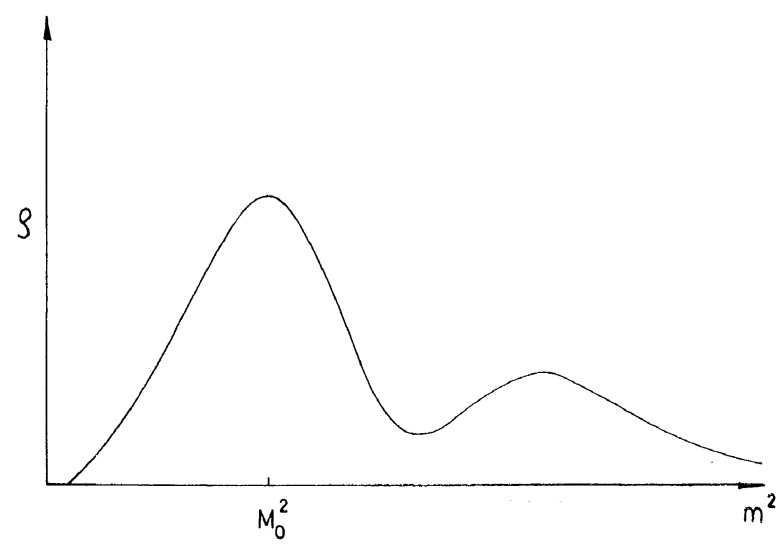

Fig. 1. 


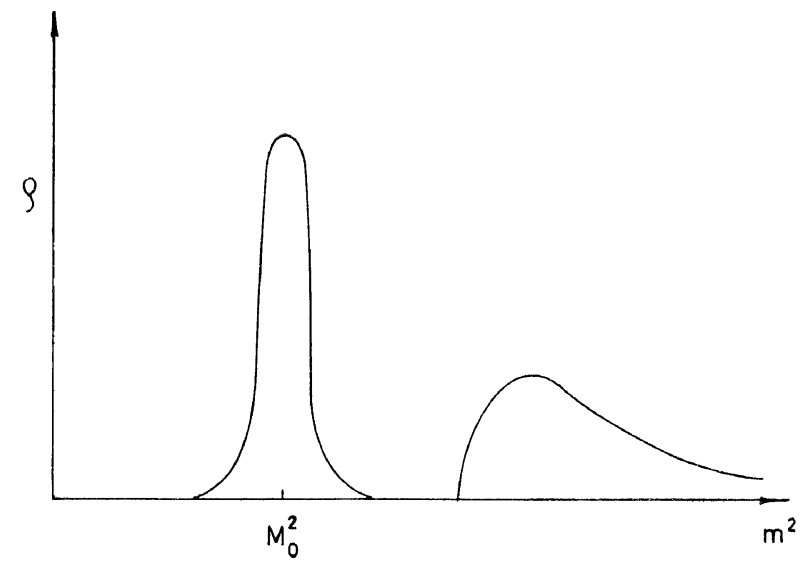

Fig. 2.

weak interaction it would be a stable particle. Therefore, there would be a gigantic hump which you might have to draw up as high as the moon on your scale, corresponding to the strong interaction, corresponding to the decay of the $\Lambda^{\circ}$ into a proton and a $K^{-}$. That you certainly do not want to include.

Salam: The probability for a $\Lambda^{\circ}$ going into a proton and two $\pi$ mesons will be equal to

$$
\left[\frac{G_{\text {weak }}}{G_{\text {strong }}}\right]^{2} \text {. }
$$

This is about $10^{-12}$. One in $10^{12} \Lambda^{\circ}$ s will decay into a proton and two $\pi$ mesons, provided the experimentalist is willing to call such an object a $\Lambda$-particle.

Gell-Mann: Let me continue what I was saying. You will have an enormous hump corresponding to the $\Lambda^{\circ}$ going into a $K$ and a proton. Of course, this is for energies far above that of the $A^{\circ}$. This, of course, you do not want to include in your sum.

Salam: No.

Gell-Mann: Have you defined clearly which set of states you are going to sum over? Presumably you will include only those states which violate strangeness conservation.

Salam: That, for example, would be a good definition. Nobody has discovered how to isolate the resonances in nuclear physics, except when you have selection rules of the type given by strangeness violation.

Gell-Mann: But this is the problem that you have attacked.

Salam: No, no. I do not claim to have attacked the problem of separating resonances when they refer to particles with the same quantum numbers.

Matthews: If you want that definition to apply to the $\Lambda^{\circ}$ you sum only over the proton + pion state.
Gell-Mann: What is the difference between proton plus pion and proton plus two pions?

Matthews: The final states consist of stable particles for which you can use the conventional Lehman - Symanzik Zimmerman procedure to define the states.

Gell-Mann: I was not aware that considering the interactions you could really distinguish between proton plus pion and proton plus two pions, since a pion incident on the proton can produce two pions.

Peierls: The point is this. Are we talking about the unstable $\Lambda^{\circ}$-particle as the experimentalist would define it, or are we talking about a physical state? Now, the $\Lambda^{\circ}$-particle has not existed for ever. It must have been created in some way. It seems to me that those curves on the board relate to what happens when you produce a particle in any of the various ways that this can be done. Then of course, you get various humps. In essence the process leading to a stable particle or an unstable particle or even to a very broad resonance look very similar. The question is: can we do something else and describe the unstable particle by itself, by a wave function? This is what Salam has called $\Phi$. He says very little about how he defines it and it is clear that it cannot be defined exactly; because it is only to the extent to which the width of such a peak is negligible compared to the distance to the neighbouring peak that you can give a meaning to such a thing.

Gell-Mann: I think there is still a hope that there might be an actual definition. That is what I expected when this report was started - a definition independent of arbitrary things. The point of view which Salam attributed to you, Peierls, sounds more likely to give a unique definition. To use a slightly different language: this $\varrho$-function is the imaginary part of what would usually be called an amplitude. If this $\varrho$-function by itself is in some way analytically continued so that the behaviour of a function reducing to $\varrho$ on the real axis can be investigated in the complex plane, I think it would be found that poles will appear off the axis and that those poles will be unique in their position. They will correspond to a particular real and imaginary part and this may some day be a real definition of the mass and lifetime of an unstable particle.

Peierls : I agree with that. It seems to me to be the right way of describing unstable particles. The reason I said this is only approximate, is because even when you get into regions of high energy where you get many broad resonances which cannot physically be described as particles you will still get lots of poles in the continuation of the propagator $G$. The question of when to call such a thing a particle and use a special function to describe it, is a quantitative one and not a qualitative one.

Salam: I would like to point out that I think Peierls agreed to something quite different from what I understood Gell-Mann is maintaining. If the propagator is 


$$
G\left(k^{2}\right)=\int \frac{\varrho\left(M^{2}\right) d M^{2}}{k^{2}-M^{2}},
$$

Gell-Mann conjectures there are poles in $\varrho\left(M^{2}\right)$ in the $M^{2}$-plane, Peierls conjectures there are poles in the "unphysical sheets" of $G\left(k^{2}\right)$, and both want the poles to be at the same place.

Gell-Mann: These two things are exactly the same.

Salam: Is there such a theorem?

Treiman: What is it the experimentalists measure? If the answer is, as it may be, that it depends on the production, then how do you divorce this discussion from the production.

Salam: You cannot. You have to write down a rather complicated formula in terms of $\varrho$ 's to take into account the production as well.

Treiman: I see no reference to the production.

Salam: The production is not simply related. If you ask me how to measure $\varrho$ most cleanly...

Treiman: In the first place the experimentalist does measure a lifetime, at least he thinks he does.

Salam: Oh, no, no! He measures nothing of the kind. In very special cases he measures what he thinks is an exponential...
Treiman: What is the connection, why is it that so many people are fooled?

Salam: If the lifetime in decay is very much larger than the lifetime in production, then one can talk about an exponential. If this is not the case then there is no experimental exponential. And I would like to challenge the experimentalist to produce one.

Gell-Mann: May I try to answer Treiman's question? In the case of a very narrow resonance such as a weakly decaying particle, the experimentalists do not get into much trouble practically. In the case of something like the $(3 / 2,3 / 2)$ resonance, which is a very broad one, certainly the answer will depend to some extent on the experimental methods. But I think that what is being sought here is the possibility that theoretically there may be a certain parameter which occurs naturally in the theory in the description of several processes which go through this same resonance. And I think that is to be found in the poles in the complex plane of $\varrho$.

Treiman: But that would have to be demonstrated.

Gell-Mann: Yes. Maybe someone has, I am not up to date on these things.

Chairman: I would propose now, the time being so late, that we close this session which has been so rich in very different reports.

\section{LIST OF REFERENCES}

1. Wataghin, G. Causality, complementarity and S-matrix formalism in a non-local relativistic theory of fields. (to be published in Nuov. Cim.) 